\title{
Influence of system geometry and bulk dielectric polarization upon PD transients
}

\author{
Pedersen, Aage; Crichton, George C; McAllister, lain Wilson
}

Published in:

IEEE 1996 Annual Report of the Conference on Electrical Insulation and Dielectric Phenomena

Link to article, DOI:

10.1109/CEIDP.1996.564530

Publication date:

1996

Document Version

Publisher's PDF, also known as Version of record

Link back to DTU Orbit

Citation (APA):

Pedersen, A., Crichton, G. C., \& McAllister, I. W. (1996). Influence of system geometry and bulk dielectric polarization upon PD transients. In IEEE 1996 Annual Report of the Conference on Electrical Insulation and Dielectric Phenomena (Vol. Volume 2, pp. 545-549). IEEE. https://doi.org/10.1109/CEIDP.1996.564530

\section{General rights}

Copyright and moral rights for the publications made accessible in the public portal are retained by the authors and/or other copyright owners and it is a condition of accessing publications that users recognise and abide by the legal requirements associated with these rights.

- Users may download and print one copy of any publication from the public portal for the purpose of private study or research.

- You may not further distribute the material or use it for any profit-making activity or commercial gain

- You may freely distribute the URL identifying the publication in the public portal 


\title{
Influence of System Geometry and Bulk Dielectric Polarization
} upon PD Transients

\author{
A. Pedersen ${ }^{\dagger}$ \\ G.C. Crichton and I.W. McAllister \\ Department of Physics Department of Electric Power Ergineering \\ Technical University of Denmark \\ DK-2800 Lyng by \\ DENMARK
}

\begin{abstract}
A physically valid theory of partial discharge transients has been developed through a field theoretical approach. The theory is based upon the concept of the charge induced upon the detecting electrode by the partial discharge. This induced charge consists of a component associated with the actual space charge in the void, and one related to changes in the polarization of the bulk dielectric. The latter is brought about by changes in the field external to the void due to the void space charge. The magnitude of the induced charge and its components are discussed with reference to a coaxial, heterogeneous dielectric-system.
\end{abstract}

\section{Introduction}

The occurrence of a partial discharge in a gaseous void leads not only to a charge being induced on the detecting electrode, but also to a change in the polarisation $\delta \vec{P}$ of the bulk dielectric. Previously the authors have shown that this change is reflected in the charge induced upon the detecting electrode $[1,2]$.

The induced charge can be evaluated using either the $\lambda$-function, or the $\phi$ function [1]. These functions account for the dielectric polarization either implicitly $(\lambda)$ or explicitly $(\phi)$. Thus by using these two functions it is

\footnotetext{
$\dagger_{\text {deceased }}$
}

possible to identify tize influence of the change in dielectric polarization upon the induced charge.

In the present paper, the influence of the system geometry upon $\delta \vec{P}$ is examined for a two dielectric system. It is shown that the component of the induced charge due to $\delta \vec{P}$ may increase or decrease depending upon the ratio of the dielectric permittivities and within which medium the void is located. The magnitude of this increase/decrease is also dependent upon the non-uniformity of the system geometry. This behaviour is reflected in the Poissonian induced charge $q$.

\section{Poissonian Induced Charge}

The induced charge can be described in terms of a Poissonian and a Laplacian component [3]. The Poissonian induced charge is that component of the induced charge which is rigidly linked to the space charge source, and which together with this source gives rise to the Basic Poisson Field [1]. Mathematically, the final value of the poissonian induced charge $q$, due to a partial discharge, can be resolved into two components:

$q=q_{\mu}+q_{p}$

where $q_{\mu}$ is the induced charge directly associated with the space charge in the void, and $q_{P}$ represents the induced charge related to the change in

(0-7083-3580-5) 1996 IEEE Annual Report - Conference on Electrical Insulation and Dielectric Phenomena, San Francisco, 0ctober 20-23, 1996 
dielectric polarization $(\delta \vec{P})$ due to the presence of this space charge [1]. With reference to the detecting electrode and the induced charge, the effect of the void wall charges can be considered as the effect of an electric dipole of moment $\vec{\mu}$ located within the void, The total poissonian induced charge arising from this dipole is given by

$q=-\vec{\mu} \cdot \vec{\nabla} \lambda$

where $\lambda$ represents the proportionality factor between the charge in the void and the induced charge on the detecting electrode. The $\lambda$-function is a solution of the general Laplace equation [1]

$\vec{\nabla} \cdot(\varepsilon \vec{\nabla} \lambda)=0$

in which $\varepsilon$ denotes permittivity. The boundary conditions are $\lambda=1$ at the detecting electrode, and $\lambda=0$ at the surfaces of all other electrodes. In addition, the following condition must be fulfilled at all dielectric interfaces such as the walls of voids

$\varepsilon_{+}(\partial \lambda / \partial n)_{+}=\varepsilon_{-}(\partial \lambda / \partial n)_{-}$

where $\lambda$ is differentiated in the direction normal to the interface, and the signs + and - refer to each side of the interface, respectively. Any method of solving Laplace's equation can be used to determine $\lambda$.

If, however, the dimensions of the void are such that $\vec{\nabla} \lambda$ may be assumed constant within the void, then we can introduce another function, $\lambda_{0}$, which represents the unperturbed $\lambda$-function in the absence of the void. As $\lambda$ is a solution of Laplace's equation, then by mathematical analogy with electrostatic fields, the relationship between the $\lambda$ and $\lambda_{0}$ functions is given by

$\vec{\nabla} \lambda=h \vec{\nabla} \lambda_{0}$
For the voids under consideration, the parameter $h$ is a scalar which depends on the void geometry and the relative permittivity of the bulk medium. Following the introduction of $\lambda_{0}$, the Poissonian induced charge on the detecting electrode may be expressed as

$q=-h \vec{\mu} \cdot \vec{\nabla} \lambda_{0}$

The component of the Poissonian induced charge related to the void space charge alone may be obtained via another proportionality factor: i.e.

$q_{\mu}=-\vec{\mu} \cdot \vec{\nabla} \phi$

where the $\phi$ function is a solution of the reduced Laplace equation [1]

$\nabla^{2} \phi=0$

The boundary conditions are $\phi=1$ at the detecting electrode, and $\phi=0$ at the surfaces of all other electrodes.

Hence, from (1), (6) and (7), the polarization component $q_{P}$ of the Poissonian induced charge may be expressed as

$q_{P}=-\vec{\mu} \cdot\left(h \vec{\nabla} \lambda_{0}-\vec{\nabla} \phi\right)$

\section{Heterogeneous Dielectric System}

The influence of $\delta \vec{P}$ upon the induced charge has previously been examined for a planar heterogeneous dielectric geometry [2]. For such a simple system, it was possible to illustrate the basic consequences associated with $\delta \vec{P}$. To extend that study, we will consider a non-uniform field geometry, i.e., a coaxial cylinder geometry with a two layer dielectric. If, in cylindrical coordinates, the inner electrode is represented by the surface $r=a$, while $r=b$ is the inner surface of the outer electrode, then $r=c$ represents the 
dielectric interface with $a<c<b$. The permittivity of the inner dielectric is $\varepsilon_{1}$ for which $a<r<c$, while that of the outer is $\varepsilon_{2}$, for which $c<r<b$. If the complete inner electrode is used to detect the partial discharge transient, then the boundary conditions for the $\lambda$-function are $\lambda_{0}=1$ for $r=a$, $\lambda_{0}=0$ for $r=b$. Hence the $\lambda_{0}$ functions of the two media are given by

$\lambda_{01}=\frac{\varepsilon_{1} \ln (b / c)+\varepsilon_{2} \ln (c / r)}{\varepsilon_{1} \ln (b / c)+\varepsilon_{2} \ln (c / a)}$

for $a \leq r \leq c$, and

$\lambda_{02}=\frac{\varepsilon_{1} \ln (b / r)}{\varepsilon_{1} \ln (b / c)+\varepsilon_{2} \ln (c / a)}$

for $c \leq r \leq b$, where the $\lambda_{0}$ subscripts, $1 \& 2$, refer to the inner and outer regions, respectively.

On differentiating with respect to $r$, we obtain the relevant expressions for the associated $\lambda_{0}$ gradients :

$\vec{\nabla} \lambda_{01}=\frac{-\left(\varepsilon_{2} / r\right) \vec{e}_{r}}{\varepsilon_{1} \ln (b / c)+\varepsilon_{2} \ln (c / a)}$

and

$\vec{\nabla} \lambda_{02}=\frac{-\left(\varepsilon_{1} / r\right) \vec{e}_{r}}{\varepsilon_{1} \ln (b / c)+\varepsilon_{2} \ln (c / a)}$

where $\vec{e}_{r}$ is a unit vector perpendicular to the axis of the coaxial system and directed away from the inner electrode.

For a homogeneous medium, $\lambda_{0}=\phi$ and thus in such a coaxial system we have $\vec{\nabla} \lambda_{0}=\vec{\nabla} \phi=\frac{-\vec{e}_{F}}{r \ln (b / a)}$
Both (12) and (13) reduce to this expression for $\varepsilon_{1}=\varepsilon_{2}$.

\section{Induced Charge Component Due To $\delta \vec{P}$}

To undertake a comparative assessment of the influence of the dielectric geometry upon PD transients we will consider that the dipcile $\vec{\mu}$, associated with the charge which has accumulated at the void wall, is as sumed a constant in this study. Furthermore, it will be assumed that the void is more than 10 times its greatest Iinear dimension from the dielectric interface, such that the $\vec{\nabla} \lambda$ distribution within the void is effectively uniform: $i . e$. the existence of the interface does not perturb $\vec{\nabla} \lambda$ in the void. This assumption implies that the concept of $h$ is valid and that (5) may be employed.

With respect to the component of the induced charge related to $\delta \vec{P}$, we have upon combining ( 9 ) and (6)

$\frac{q_{P_{n}}}{q_{n}}=\frac{-\vec{\mu} \cdot\left(h_{n} \vec{\nabla} \lambda_{0 n}-\vec{\nabla} \phi\right)}{-\vec{\mu} \cdot h_{n} \vec{\nabla} \lambda_{0 n}}$

where $q_{n}$ is the total Poissonian induced charge of the heterogeneous system, with $n=1,2$ depending in which dielectric medium the void is located.

Owing to the cylindrical geometry, the dipole moment is either directed away from or towards the axis of the system. Consequently, this noment can be expressed as

$\vec{\mu}= \pm \mu \vec{e}_{r}$

Hence upon undertaking the vector operations, (15) reduces to

$\frac{q_{P n}}{q_{n}}=1-\frac{\mathrm{d} \phi / \mathrm{d} r}{h_{n} \mathrm{~d} \lambda_{0 n} / \mathrm{d} r}$ 
Thus using (12), (13) and (14) we obtain for a void in medium 1

$\frac{q_{P 1}}{q_{1}}=1-\frac{\left(\varepsilon_{1} / \varepsilon_{2}\right) \ln (b / c)+\ln (c / a)}{h_{1} \ln (b / a)}$

and for medium 2

$\frac{q_{P 2}}{q_{2}}=1-\frac{\ln (b / c)+\left(\varepsilon_{2} / \varepsilon_{1}\right) \ln (c / a)}{h_{2} \ln (b / a)}$

It should be noted that both (18) and (19) contain $h$. This implies that $q_{P_{n}}$ is dependent upon the void geometry. To simplify we will assume the void to be spherical, in which case we have

$h=\frac{3 \varepsilon_{r}}{1+2 \varepsilon_{r}}$

where $\varepsilon_{r}$ is the relative permittivity of the dielectric containing the void.

The variation of $q_{P n} / q_{n}$ with $\varepsilon_{2} / \varepsilon_{1}$ is shown in Figure 1 for $\varepsilon_{r}=4, s / d=0.5$ and several values of $H d$, where $H$ is the mean curvature of the inner electrode:

$H=\frac{1}{2 a}$

and $d$ represents the gap length, viz.

$d=b-a$

The value of $H d$ is a measure of the geometric non-uniformity: i.e. $H d=0$ represents a planar electrode system [2]. The parameter $s$ represents the thickness of the dielectric bounding the detecting electrode, see[2], i.e. in this instance

$s=c-a$

From Figure 1 it is seen that $q_{P_{n}}$ is increased when the void is located in the medium of lesser permittivity. Conversely a decrease occurs when located in the medium of greater permittivity. This reduction can be of such a degree that the polarity of $q_{P n}$ is reversed.

When the void is in the inner medium, i.e. for $n=1$ and $a \leq r \leq c$, the greater the Hd-value, the smaller are the relative increases and reductions in $q_{p n 1}$. The reverse behaviour occurs when the void is in the outer medium; i.e. for $n=2$ and $c \leq r \leq b$.

As $q_{P_{n}} / q_{n}$ exhibits symmetry with respect to $\varepsilon_{2} / \varepsilon_{1}=1$ for a planar geometry $(H d=0)$, see Figure 1 and [2], the asymmetrical behaviour of $q_{P n} / q_{n}$ for the coaxial geometry ( $H d>$ $0)$ is a consequence of the nonuniform geometry.

For a fixed void location, $q_{\mu}$ is dependent only on $\vec{\mu}$, which we assumed constant. Hence variations in $q_{P}$ will be reflected directly in the magnitude of the Poissonian induced charge $q$, see (1).

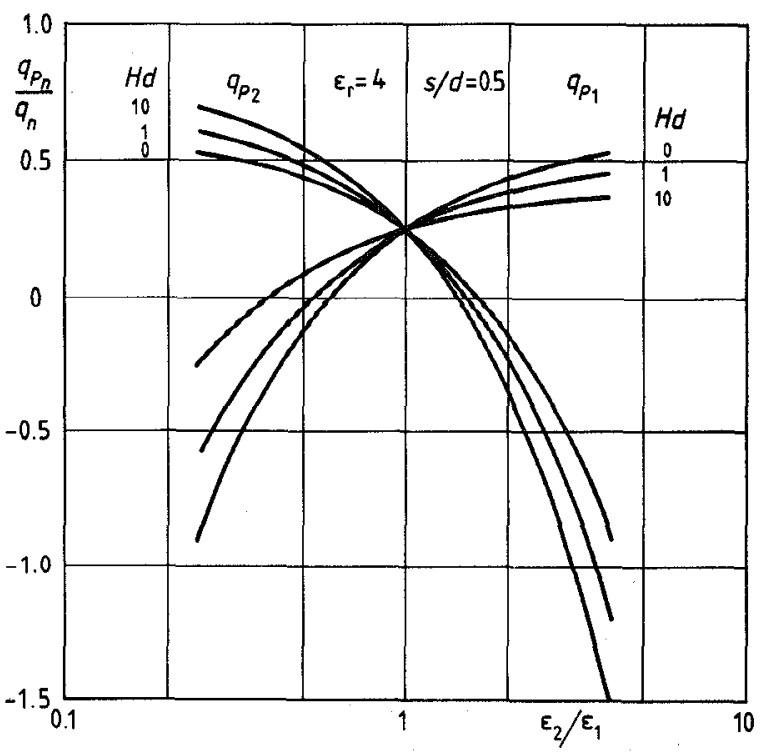

Fig. 1 Variation of the polarization component of the Poissonian induced charge. 


\section{Conclusion}

It is demonstrated that changes in dielectric polarization arising from a partial discharge can significantly affect the magnitude of the Poissonian induced charge. For a two dielectric system, this influence is dependent upon the ratio of the dielectric permittivities and within which medium the void is located. Furthermore, changes in the geometry of the system are seen to influence the polarization component. Hence the quantitative interpretation of partial discharge transients in terms of discharge phenomena within a void becomes extremely complicated in the presence of heterogeneous dielectric systems bounded by electrodes of non-zero curvature.

\section{References}

[1] A. Pedersen, G.C. Crichton and I.W. McAllister, "The Functional Relation Between partial Discharges and Induced charge", IEEE Trans. Diel. \& Elect. Insul., Vo1.2, pp.535-543, 1995.

[2] A. Pedersen, G.C. Crichton and I.W. McAllister, "Influence of Bulk Dielectric Polarization upon PD Transients", CrIDP pp.323-326, 1995.

[3] A. Pedersen, G.C. Crichton and I.W. McAllister, "Partial. Discharge Detection: Theoretical and Practical Aspects', IEE Proc. Sci. Meas. Technol., Vol.142, pp.29-36, 1995. 